\title{
Correlation Functions and Confinement in Scalar QCD
}

\section{Tajdar Mufti ${ }^{* \dagger}$}

E-mail: tajdar.mufti@uni-jena.de

\section{Axel Maas}

E-mail: axelmaas@web.de

Institute of Theoretical Physics, Friedrich-Schiller-University Jena, Max-Wien-Platz 1, D-07743 Jena, Germany

\begin{abstract}
The complete knowledge of a theory is encoded in its correlation functions. Thus non-perturbative effects, like confinement in QCD, is necessarily contained in them. As a consequence, a number of confinement scenarios make predictions for the behavior of these correlation functions. Nonperturbative methods, like lattice calculations, permit to calculate them and test these predictions. To avoid the entanglement with chiral symmetry breaking and the costs of full fermion simulations, here scalar QCD will be used as a role model, as it is expected that confinement operates in the same way. We present results on both two-point functions and three-point functions for the case of two colors and two flavors of scalars, and compare them to the predictions.
\end{abstract}

The 2013 European Physical Society Conference on High Energy Physics,

18-24 July, 2013,

Stockholm, Sweden

\footnotetext{
* Speaker.

† Supported by the DFG graduate school 1523-1 and under grant number MA 3935/5-1

$¥$ Supported by the DFG under grant number MA 3935/5-1
} 
Confinement in the loose sense of the absence of quarks and gluons as free particles is a longstanding challenge. In principle, this is resolved by axiomatic field theory, which shows that no gauge-invariant state with the quantum numbers of quarks and gluons can be defined. However, how this manifests itself in the correlation functions encoding the contents of the theory is far less clear. But this is of central importance for the description of observables using functional methods, where the gauge-dependent correlation functions serve as intermediate steps [1]. Furthermore, though confinement in the strict sense of a finite string tension is absent in QCD, it manifests itself as a residual effect, e. g. in the form of Regge trajectories.

Lattice calculations can be used to determine the correlation functions, and resolve these questions. To avoid the cost of fermions, scalar QCD is used here, as it is expected to manifest the same confining properties, without the complications due to chiral symmetry, as long as it assumed that possible triviality issues do not interfere at low energies. A more severe problem is that there is no gauge-invariant distinction between a would-be Higgs phase and a would-be confinement phase of the theory [2], at least on a finite lattice. However, it turns out that the gauge-invariant spectrum of the theory has quite distinct properties [3, 4]. In the would-be QCD phase the lightest excitation is a flavor-singlet $0^{++}$state, while in the would-be Higgs phase it is a $1^{--}$flavor triplet, at least for two flavors. This observation will serve here as an operational definition of what is meant by scalar QCD, using only the region where the scalar is a much lighter state than the vector state.

This provides a setup where the correlation functions of the theory can be calculated on the lattice, here in minimal Landau gauge [1]. Details of the calculations can be found elsewhere [3,5]. The scale for these calculations is set such that the lightest state has a mass of $1 \mathrm{GeV}$, inspired by the scale set by the proton.

For the two-point function it is found [3] that the gauge sector behaves as in Yang-Mills theory [1], while the matter sector is similar to the quenched case [6]. Since the vertices show a much more intriguing behavior, details on the two-point functions will be relegated to [3].

Our results for the three-point tree-level dressing functions are shown in figure 1. While the gauge sector vertices of three gluons and of a gluon, a ghost, and an anti-ghost behave like in the Yang-Mills case [1], the scalar-gluon vertex shows within the larger errors no deviations from tree-level, especially no infrared singularities which would yield a confining potential already from (quasi-)one gluon exchange [7], in contrast to the expectation [8], but in line with the existence of alternative solutions [9]. This implies that, at least in minimal Landau gauge, possibly infinite sums of correlation functions are required to create the intermediate-distance string tension. However, this does not imply yet that Regge trajectories could not already be encoded in the four-point functions alone.

\section{References}

[1] A. Maas, Phys. Rep. 524, 203 (2013), 1106.3942.

[2] E. H. Fradkin and S. H. Shenker, Phys. Rev. D19, 3682 (1979).

[3] A. Maas and T. Mufti, unpublished.

[4] J. Jersak, C. B. Lang, T. Neuhaus, and G. Vones, Phys. Rev. D32, 2761 (1985). 

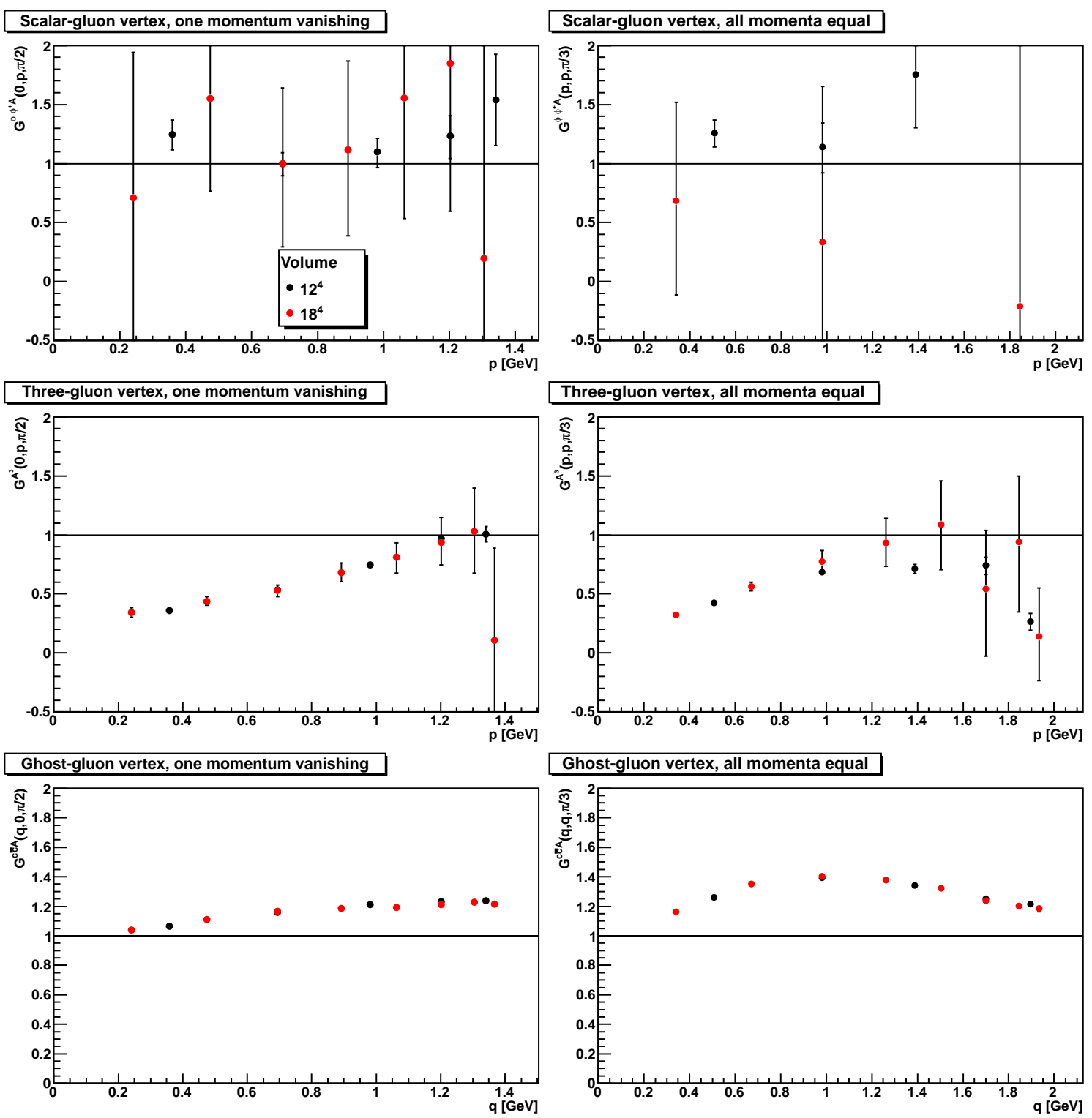

Figure 1: Dressing functions of the tree-level tensor structure [1] of the scalar-gluon vertex (top panel), three-gluon vertex (middle panel), and ghost-gluon vertex (bottom panel). The straight line is the tree-level value. The lattice spacing is $a^{-1}=694 \mathrm{MeV}$, and at tree-level the scalar quarks are massless and have no self-interactions.

[5] A. Maas, Mod.Phys.Lett. A28, 1350103 (2013), 1205.6625.

[6] A. Maas, PoS FacesQCD, 033 (2011), 1102.0901.

[7] R. Alkofer and L. von Smekal, Phys. Rept. 353, 281 (2001), hep-ph/0007355.

[8] L. Fister, R. Alkofer, and K. Schwenzer, Phys. Lett. B688, 237 (2010), 1003.1668.

[9] C. S. Fischer and J. M. Pawlowski, Phys. Rev. D80, 025023 (2009), 0903.2193. 